\title{
A Qualitative Methodology to Support the Evaluation of Novel Treatments for Hyperphagia in People with Prader-Willi Syndrome
}

\author{
Mindy Leffler, MEd ${ }^{1}$, Sonya J Elder, PhD ${ }^{1^{*}}$, Siri Bolding, $P h D^{1}$, Megan Hefner, $M S^{1}$, Jennifer $L$ Miller,

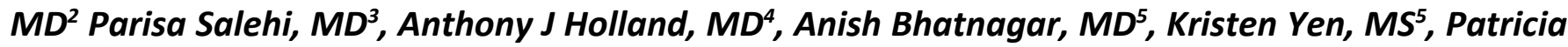 \\ C Hirano, $\mathrm{MPH}^{5}$ and Kristina Davis, PhD $^{1}$
}

${ }^{1}$ Casimir, Plymouth, USA

${ }^{2}$ Department of Pediatric Endocrinology, University of Florida, USA

${ }^{3}$ Division of Endocrinology, Seattle Children's Hospital, University of Washington, USA

${ }^{4}$ University of Cambridge, Cambridge, UK

${ }^{5}$ Soleno Therapeutics, Redwood City, USA

*Corresponding author: Sonya Elder, PhD, Research Scientist, Casimir, 36 Cordage Park Circle, S 300, Plymouth, MA 02360, USA, Tel: 2062-508-346

\begin{abstract}
Title: A Qualitative Methodology to Support the Evaluation of Novel Treatments for Hyperphagia in People with PraderWilli Syndrome.

Background: Assessing change in symptoms affecting people with Prader Willi Syndrome (PWS), a rare disease, is complicated by the influence of different levels of food security procedures and the impact of immediate circumstances on symptom presentation and severity. We report on the use of qualitative interviews to collect information on behavioral change and on the impact of factors specific to individual participants with PWS in a clinical trial.

Methods: Soleno Therapeutics' Phase 3 program consists of a double-blind study, an open-label extension study as well as a sub-study consisting of qualitative interviews collected at baseline, the end of the randomized period, and two points during the open label extension. Interviews were conducted with a semi-structured guide covering specific food-security procedures and three areas of interest: foodrelated behavior, non-food-related behavior, and aspects of daily life. Two coders trained in qualitative analysis singlecoded baseline interview transcripts with a dual-coded subset to confirm reliability. From follow-up interviews mentions of change will be dual-coded, with coders discussing any discrepancies to reach resolution. In future analyses, descriptions of change that also include potential attributions from caregivers will include that contextualization based on a pre-specified adjudication plan.
\end{abstract}

\begin{abstract}
Results: The analysis of the baseline interviews identified a variety of food-security procedures practiced by families of people with PWS participating in the clinical trial, ranging from total lockdown since diagnosis to the absence of any food security or routines. Behaviors that were the subject of the qualitative interview were present at baseline in numbers varying from 17 to $100 \%$ of the participants in this study. Specific behaviors reported present in all trial participants varied in their manifestation, for example, frequent conversations about food were described by some families as a negative symptom (arguments, nagging, manipulation) and by others as a positive (productive discussion of food choices).
\end{abstract}

Conclusion: The heterogeneity of the trial participants in the studies of DCCR in people with PWS, as evidenced by the baseline qualitative interviews, supports the need to obtain a further understanding of trial participantspecific experiences before and during a clinical trial. This approach could be used in clinical trials to help support and contextualize treatment efficacy in rare disease populations when used alongside other clinical outcomes.

\section{Keywords}

Prader-Willi Syndrome, Clinical trials

Abbreviations
PWS: Prader-Willi Syndrome; RCT: Randomized, Controlled
Trial; OLE: Open-Label Extension; HQ-CT: Hyperphagia
Questionnaire for use in Prader-Willi Syndrome Clinical
Trials; DCCR: Diazoxide Choline Controlled Release

Citation: Leffler M, Elder SJ, Bolding S, Hefner M, Miller JL, et al. (2021) A Qualitative Methodology to Support the Evaluation of Novel Treatments for Hyperphagia in People with Prader-Willi Syndrome. Int J Rare Dis Disord 4:030. doi.org/10.23937/2643-4571/1710030

Accepted: June 28, 2021; Published: June 30, 2021

Copyright: (C) 2021 Leffler M, et al. This is an open-access article distributed under the terms of the Creative Commons Attribution License, which permits unrestricted use, distribution, and reproduction in any medium, provided the original author and source are credited. 


\section{Introduction}

Prader-Willi Syndrome (PWS) is a genetic disorder estimated to affect 1 in 10,000 to 20,000 individuals [1]. PWS has a complex presentation of symptoms, involving behavior, sleep, appetite, growth, sexual development, cognition, and metabolism [1-3]. PWS is characterized by an evolution from difficulty with feedings, failure to thrive, and hypotonia at birth to lower metabolic rate and hyperphagia in childhood. Individuals with PWS can also have intellectual disability, social impairments, and behavioral difficulties [2,4]. Individuals with PWS can experience multiple comorbidities including delayed puberty, short stature, diabetes mellitus, and major mental illness, including depression, especially without appropriate medical treatment [1]. Current approaches to managing PWS are aimed at managing specific symptoms, such as using growth hormone to treat short stature, controlling access to food to prevent life-threatening obesity, and interventions and support strategies for improving well-being and behavior [2,5].

Hyperphagia is the hallmark symptom of PWS, involving food-seeking, food-related anxiety, and lack of self-control around food $[4,6]$. Other common behavioral disturbances in PWS are difficulty coping with change, lack of age-typical emotion regulation, anxiety, and temper outbursts, lack of impulse control, manipulative behavior, obsessive compulsive tendencies, and skin picking. Some of the behaviors seen in PWS overlap with those seen in autism spectrum disorder [7]. The presentation and severity of each symptom can vary greatly within the PWS population $[3,6,7]$. Studies of underlying genetics reveal a tendency towards behavioral profiles within genetic subtypes rather than a clear delineation between profiles $[1,3,4,8]$. In addition, the behavioral profile of the PWS population changes across the lifespan $[3,6,8]$. Powering a clinical trial may require including a broad age range of participants with PWS to reach the required numbers, contributing to the symptom heterogeneity of the study population and potentially conflating treatment-related change with maturational change. Age-related variability within a study may also be related to the evolution of standards of care for people with PWS, with only younger participants having benefitted from recently defined care standards and greater use of growth hormone from early childhood.

Current standards of care specify the importance of a structured and controlled living environment to reduce anxiety and food insecurity $[2,6,7,9]$. The structure is often described to caregivers as 1) No hope, restricting access to food using locks or other deterrents, 2) No doubt, instituting a food routine setting expectations and 3) No disappointment, reminding caregivers not to make promises that are not fulfilled [9]. Supporting people with PWS in a secure food environment is postulated to have downstream effects on other problem behaviors by reducing anxiety and focus on food [10].
People with PWS have a need for structure and often respond negatively to changes to routine [7]. In addition, symptom presentation and severity in people with PWS worsens with stress [9]. Thus, historical living environment and immediate circumstances may influence symptom profile and function.

The heterogeneity of the PWS population combined with the impact of environmental context challenges the assumption that a therapeutic effect will look similar between participants with PWS in a clinical trial. Understanding the impact of changes to a trial participant's immediate context (moving households, initiating a new therapy or routine, quarantining due to a global pandemic) and historical context (food security and routines) are important for interpreting changes reported during a clinical trial. Additionally, changes to routine or additional stressors may have a deleterious effect on the behavior of a trial participant with PWS that could appear to be a worsening of symptoms and could be falsely attributed to the agent being evaluated. Clinical trial participation, particularly in rare disease, often requires travel, resulting in a change in routine, introduction of unfamiliar people, and movement from a structured home environment; the changes from the home environment that have an impact on quality of life may not be represented during in-clinic assessments in a trial [11].

Soleno Therapeutics (Soleno) is sponsoring a Phase 3 clinical program of DCCR (Diazoxide Choline) extended-release tablets to treat hyperphagia in people with PWS. This program comprises a 13-week Phase 3, randomized, double-blind, placebo-controlled study (C601 or DESTINY PWS; NCT03440814) with an open label extension (OLE) study (C602) for up to 36 months. During the trial, 127 participants with PWS (ages 4 years and older) with genetically-confirmed PWS were randomized at 29 sites in the US and UK. The primary endpoint in DESTINY PWS was change in hyperphagia from baseline assessed using the Hyperphagia Questionnaire for Clinical Trials (HQ-CT), a caregiver-reported assessment [7]. The secondary endpoints in DESTINY PWS included body fat mass change, Clinical Global Impression of Improvement and Caregiver Global Impression of Change.

The use of caregiver questionnaires to assess the effectiveness of a trial drug for hyperphagia in people with PWS is necessary since PWS subjects are themselves unable to reliably respond to questions, but it also has challenges. For instance, the HQ-CT has frequency ("How often..." or "How much...") and Likert scale ("Not at all" to "Extremely") questions but does not collect historical or immediate context in support of those assessments. FDA guidance on patient-focused drug development advises that interviews within the context of a clinical trial can help identify symptom changes, participant experiences, preferences, motivations, and expectations, and add greater depth to data in trials involving peo- 
ple with rare disease [12]. Conducting qualitative interviews with caregivers during a clinical trial may address the current measurement challenges that existing PWS outcome measures face. Considering the potential importance of symptom heterogeneity and contextual influences on the assessment of treatment outcomes, a sub-study was designed to co-occur with DESTINY PWS and the OLE in which caregivers of trial participants with PWS participated in semi-structured qualitative interviews to document change in a participant-specific way that included context. This paper reports on the concept elicitation work performed, the planned analysis for the baseline, DESTINY PWS and OLE study data, and the results of the baseline heterogeneity analysis.

\section{Methods}

\section{Study design}

The optional sub-study (NCT04102839) sponsored by Soleno Therapeutics and conducted by Casimir consisted of semi-structured, qualitative audio/video interviews with caregivers of participants with PWS enrolled in the DCCR studies. Interviews were conducted remotely at the start and end of the double-blind period and at 13-15 weeks and 52-54 weeks into the OLE. Interviews occurred after key clinic visits to avoid introducing bias into in-clinic assessments. The sub-study protocol was IRB/IEC-approved prior to initiating any sub-study procedures, and appropriate informed consent and assent were obtained from all caregivers and participants.

\section{Interview guide development}

Development of the interview guide included a literature review and a series of preliminary, unstructured, concept elicitation interviews conducted in parallel with caregivers and clinicians directly involved in the care of people with PWS. A total of six families participated in the interview guide development, representing four males and two females with PWS between eight and 25-years-old. Five clinicians participated, two in the UK and three in the US. The interviews focused on identifying aspects of the disorder meaningful in the context of this Phase 3 program that may not be fully captured by current outcome measures. The interviews also explored the symptom severity spectrum, the manifestation of symptoms amongst participants, and the structure of each family's home environment. After the concept elicitation interviews were conducted, the caregivers followed by the clinicians were given a chance to review a draft of the interview guide. Three of the children with PWS were raised with complete food security from an early age, while three only had moderate food security measures in place.

The concept elicitation interviews highlighted a range of symptom severity and manifestations (Figure 1).

In this small sample, the relationship between food security in the home and symptom severity was complex; some narratives suggested that a more secure environment resulted in less hyperphagia, while others described a person with PWS with enough self-control that strict food security measures were unnecessary.

The range of responses in concept elicitation interviews suggested a degree of underlying heterogeneity and a complex interaction with environment warranting the addition of this sub study to the Phase 3 program. It was also clear that multiple interviews would be needed over the duration of the program to appropriately capture potential drug effects. Semi-structured interview guides were developed to broadly introduce behaviors and allow caregivers to expound on their specific observations through open-ended answers. The guide was structured into five sections: Background, Food Behavior, General (non-food-related) Behavior, Daily Life, and Impact on Caregiver.

\begin{tabular}{|l|l|l|}
\hline $\begin{array}{l}\text { Is not opportunistic about food } \\
\text { Hyperphagia is less of an impact } \\
\text { than rigidity of meal timing }\end{array}$ & $\begin{array}{l}\text { Never feels full; constantly } \\
\text { driven by food }\end{array}$ \\
\hline $\begin{array}{l}\text { Will take a nap or self-isolate if } \\
\text { emotions escalate }\end{array}$ & Behavior \\
and is always food seeking
\end{tabular}

Figure 1: Range of behaviors reported during concept elicitation in key domains. 


\section{Study population and recruitment}

DCCR study site personnel obtained permission to share caregiver contact information with Casimir; interested caregivers were contacted by Casimir staff for consent and enrollment in the interview sub-study. Caregivers of trial participants could enroll in the substudy at any point during the RCT or OLE study and participated in interviews from that time point to the end of the study. In this report, 'caregiver' refers to the person supporting an individual with PWS who were recruited for this qualitative study. The terms 'participant' or 'trial participant' refers to the individuals with PWS recruited into the DESTINY PWS and OLE trials.

\section{Interview procedures}

Interviews were conducted by Casimir staff trained in qualitative research. All interviews were conducted remotely on a HIPAA- and GDPR-compliant web conferencing platform. The interviews lasted approximately one hour, and caregivers were compensated for their time. Caregivers could opt for interviews to be recorded as video and audio or audio only. Audio/video files were transcribed verbatim, and transcripts were checked for accuracy by Casimir staff.

During follow-up interviews, caregivers were asked if changes were noticed in all behaviors discussed and about their perceptions of change, including timing. Meaningfulness of change was assessed through domain-specific questions: "Have you noticed changes in your child's food behavior/behavior/daily life?" If change was noticed, caregivers were asked to classify the change as positive or negative and as having a major or minor impact on quality of life. Follow-up interviews are currently in progress and will be discussed in a subsequent publication.

\section{Analysis}

Two coders trained in qualitative analysis developed an independent list of themes based on the concept elicitation interviews and refined by review of the baseline transcripts. The two coding lists were then reconciled and structured into a master codebook. All coding was completed using NVivo 13 software (QSR International) by blinded coders. Transcripts were single-coded for themes, with a dual-coded subset to ensure reliability among coders. Domain-specific change items and any mention of change in follow-up interviews were dual-coded, with coders resolving any discrepancies. To minimize potential bias, coding was conducted only by blinded Casimir researchers and with no participation from Soleno.

\section{Baseline data}

Baseline heterogeneity was examined through aggregated frequencies with which each theme/behavior was reported. Additionally, baseline heterogeneity was summarized in narrative form to report qualitative sim- ilarities or differences among participants with similar behaviors.

\section{Results from Baseline Assessments}

\section{Cohort - age and gender}

The cohort included caregivers of 12 participants with PWS who participated in a baseline interview prior to Visit 3 of the DESTINY PWS study (mean age of participants $11+/-5.3$ years, range 6-25). In general, male participants were older (mean $15+/-7.2$, range: 8-25) than females (mean $9+/-2.8$, range: 6-14). Of the 12 participants, 8 (67\%) were female.

\section{Food security}

Level of food management and food security measures ranged widely. Half of caregivers kept locks on food storage doors. Eleven caregivers described following some food schedule with one reporting locked food access and close monitoring but no food schedule. Seven caregivers reported a strict schedule, along with close monitoring/supervision. Two caregivers reported a combination of clear expectations and schedule without locks or close supervision. One caregiver reported near constant negotiations related to food and did not mention any routine or schedules.

Differences in food security were apparent in caregivers' descriptions of daily life practices. For instance, the caregiver of a 9-year-old female kept to a strict routine because her daughter "really is most at peace if I'm on top of having all her containers labeled, ready to go." Other participants did not require restrictive security measures; the caregiver of an 8-year-old female reported she was "not locking the fridge yet. Just because we did not get the lock, I guess. But I'm watching her $24 / 7$. So it's like she's never... five minutes that we don't watch her in the house." In contrast, a 13-year-old male participant broke the key-based locks, necessitating a change to keypad locks that were less vulnerable to his attempts to circumvent the security measures.

\section{Food-related behavior}

A summary of food-related behaviors can be found in Table 1 and in Figure 2. In Table 1, $\mathrm{N}$ was less than 12 for some behaviors because some caregivers did not address particular topics.

Although some food-related behaviors were present for each family, the nature and frequency varied. All 12 caregivers reported frequent conversations about food. For three participants, most conversations related to food planning/preparation, and participants were generally amenable to caregiver suggestions. For six participants, food conversations were described as negotiations including repetitive questions, asking for seconds, and bargaining; some (two) of the negotiations were associated with tantrums. In two homes, contentious conversations around food were reported as common, 


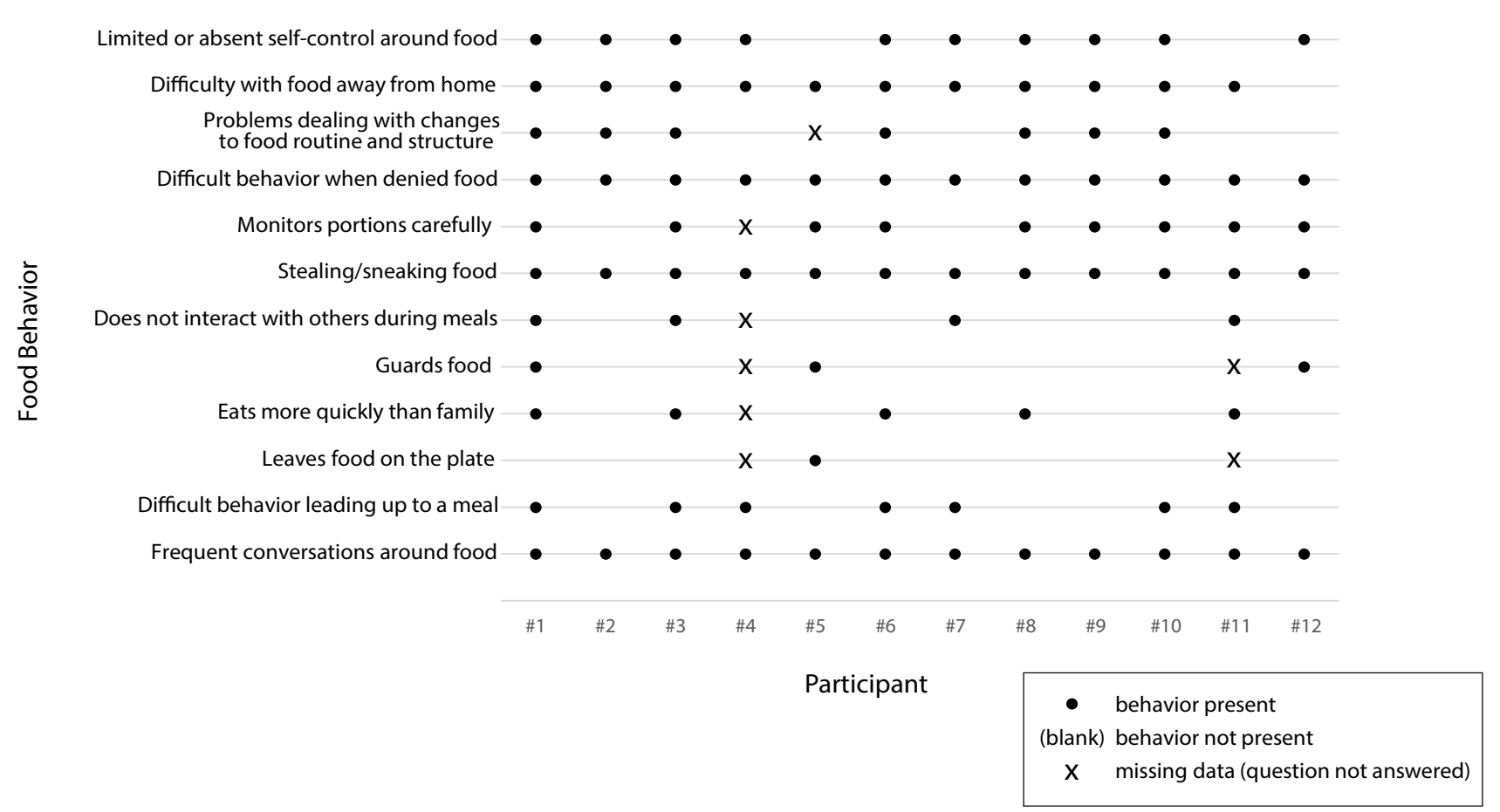

Figure 2: Food Behavior by Participant at Baseline.

Table 1: Summary of baseline food-related behaviors.

\begin{tabular}{|l|l|}
\hline Behavior & Subjects n/N (\%) \\
\hline Frequent conversations about food & $12 / 12(100)$ \\
\hline Difficult behavior leading up to a meal & $7 / 12(58)$ \\
\hline Leaves food on the plate & $1 / 10(10)$ \\
\hline Eats more quickly than family & $5 / 11(45)$ \\
\hline Guards food & $3 / 10(30)$ \\
\hline Does not interact with others during meals & $4 / 11(36)$ \\
\hline Stealing/sneaking food & $12 / 12(100)$ \\
\hline Monitors portions carefully & $9 / 11(82)$ \\
\hline Difficult behavior when denied food & $12 / 12(100)$ \\
\hline $\begin{array}{l}\text { Problems dealing with changes to food } \\
\text { routine and structure }\end{array}$ & $7 / 11(64)$ \\
\hline Difficulty with food away from home & $11 / 12(92)$ \\
\hline Limited or absent self-control around food & $10 / 12(83)$ \\
\hline
\end{tabular}

where the child was aggressive and prone to tantrums or acting out and/or argumentative. In one case, the caregiver described food conversations as varying, including amenable planning, negotiations, and argumentative interactions.

The behavior of the people with PWS during meals also varied greatly. Very few of the participants left food on the plate (1 of 10) or guarded food (3 of 10); some participants ( 4 of 11 ) were described as not interacting with family members during meals, and nearly half of participants with PWS were described as eating more quickly than family members (5 of 11 ). The time leading up to a meal was often problematic, but the nature of the behavior ranged from the repetitive questioning of a 14-year-old male: "He is asking every potential ques- tion about the meal. 'What are we going to eat? Where are we going to eat? What am I going to have? When exactly are we going to go?'" to the anxious behavior of a 7-year-old female: "It's like she gets anxiety about it because... she'll stand in the kitchen while you're cooking."

All caregivers reported their children would steal or sneak of food, but the severity and frequency varied. One caregiver said it seldom occurred because the child was constantly supervised. One caregiver reported their child hoarded stolen food weekly, two participants predominantly stole food when outside of the home, four participants stole/snuck food whenever an opportunity presented itself, and one participant snuck out of the house to spend his money on food. Three of the caregivers said their children took food from the garbage.

Portion monitoring by the individual with PWS was specifically described by 9 of 11 caregivers. In six cases, caregivers reported the individual with PWS getting angry, acting out, or having tantrums when portions are deemed unfair. Three caregivers reported negotiations were required regarding fair portions. One caregiver reported their child closely monitored the portioning across family members. The two participants with PWS who did not portion monitor were two of the youngest (6 and 8-years-old).

All participants with PWS experienced difficulty when denied a requested food. However, the caregivers described a range of reactions from temporary pouting to instigation of violence. A majority of caregivers ( 7 of $11,64 \%)$ described the person with PWS having difficulty coping with changes to food routine. A majority (92\%) 
also reported difficulty with food-related behavior away from home, particularly parties, restaurants, and grocery stores. Behavior away from home was influenced by preparation ahead of time, as described by the caregiver of a 9-year-old female: "I have to really make it clear to her and think always ahead of time." This behavior was also impacted by the availability of food, as described by the caregiver of a 13-year-old male: "The whole issue is, like, if you go somewhere where there's unrestricted access to food." In addition, the degree to which behavior outside of the home might be responsive to change depends on the family's willingness to take the person with PWS outside of the controlled environment.

Self-control around food was described as present in two trial participants with PWS, limited for six, and completely absent for four. Notably, the three participants with PWS described as completely lacking in self-control were also described as being particularly developmentally or cognitively disabled (e.g., 6-year-old with cognitive and processing delays, 8-year-old still not toilet trained, and 11-year-old with developmental delay). On the two extremes of the continuum, the caregiver of a 6-year-old female described her daughter's lack of self-control around food, saying "With food there really is no control... It used to be we could have like some appetizers out... it's really hard to do that now. She wants to be really close to the food." The caregiver of a 25-year-old male described her son as having a "tremendous amount" of self-control prior to the study, detailing, "He actually has been really monitoring his weight lately, and he's trying to lose weight. And so there are days where... he'll just completely skip lunch and not have anything, which I really couldn't do probably, or have a bowl of oatmeal for dinner and nothing else."

The most-problematic food-related behaviors varied and included sneaking/stealing food, behavior when denied food (outbursts, tantrums), the constant focus on and demand for food, choking resulting from fast eating, eating out with tantrums, agitation around food, and behavior when routines were disrupted.

\section{General Behavior}

A summary of selected non-food-related behaviors can be found in Table 2 and in Figure 3. In Table 2, N was 11 for argumentativeness because one caregiver did not address this topic.

Although the non-food-related behaviors were present for most or all of the participants, the nature of

Table 2: Summary of baseline non-food-related behaviors.

\begin{tabular}{|l|l|}
\hline Behavior & Subjects $\mathbf{n} / \mathbf{N}(\%)$ \\
\hline Tantrums & $12 / 12(100)$ \\
\hline Aggression & $10 / 12(83)$ \\
\hline Argumentativeness & $9 / 11(82)$ \\
\hline Anxiety & $12 / 12(100)$ \\
\hline Skin picking & $10 / 12(83)$ \\
\hline Hyper-focus & $12 / 12(100)$ \\
\hline Problems dealing with change & $9 / 12(75)$ \\
\hline
\end{tabular}

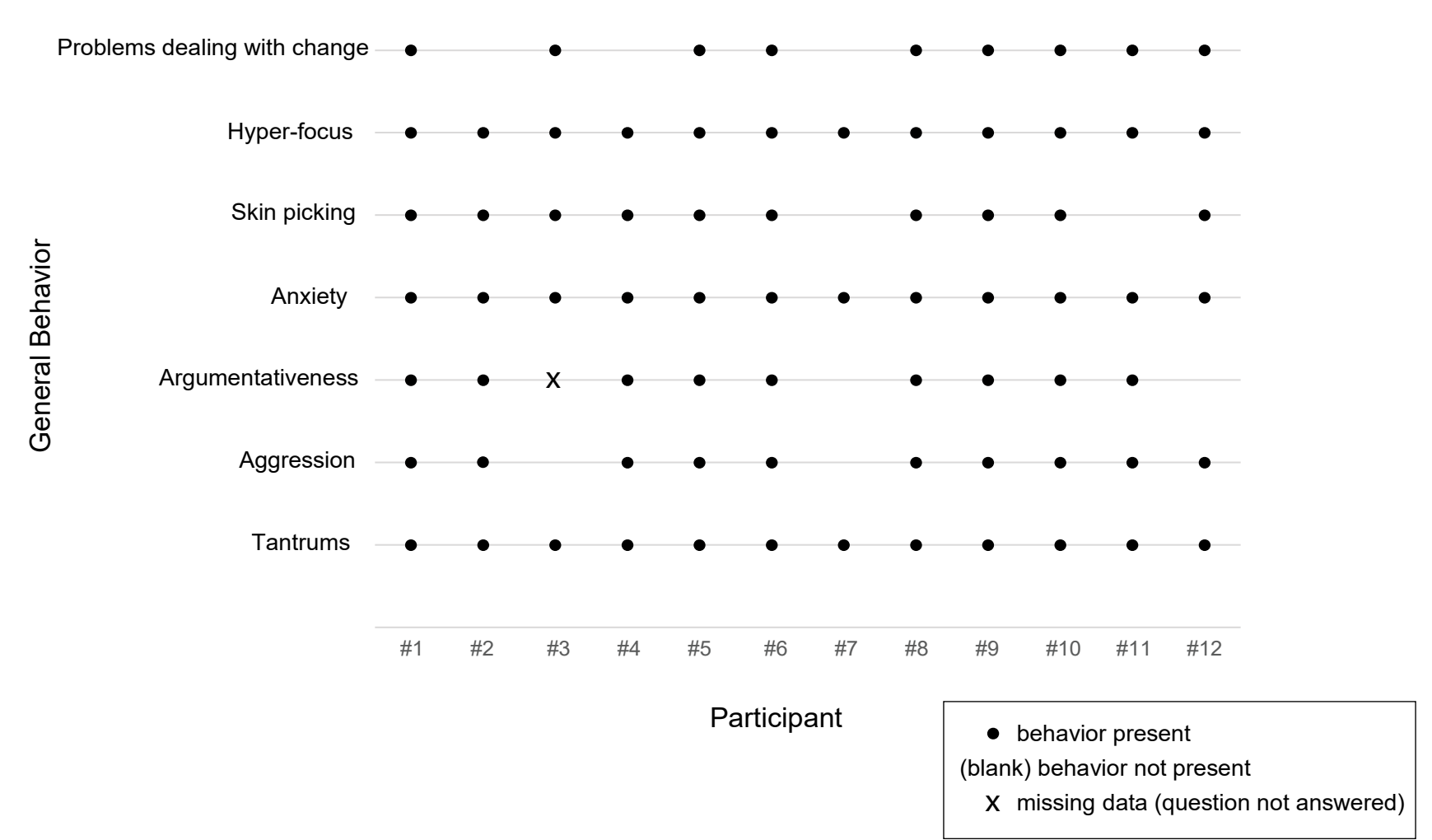

Figure 3: General Behavior by Participant at Baseline. 
these behaviors varied. All twelve caregivers reported tantrums in some form, with frequency ranging from many times per day to 2 to 3 times per year. For nine families, typical tantrums involved crying, screaming/ yelling, throwing things, and/or verbal aggression/profanity. The caregiver of a 25 -year-old male described, "They're pretty intense. So usually, he raises his voice, and then if you try to argue with him... he just yells over. You can't say anything. He's just yelling at you. He will sort of charge you and get right in your face and be screaming. He curses very nasty curses. He calls his mother very bad names and just keeps screaming them over and over..." Alternately, two caregivers reported withdrawal-like tantrums as described by the caregiver of a 15-year-old female: "A shutdown is, you know, she really kind of gets like a glassy look over her eyes, won't focus... on your face.... For extreme shutdowns she literally will just fall to the floor. She'll just noodle herself to the floor and becomes a noodle so you can't pick her up."

Four caregivers reported physical violence towards others during tantrums. The caregiver of a 14-year-old male described, "Now, I would say his tantrums over the last two years, since we've started the Celexa, have decreased in their frequency. However, they've increased in their violence." The caregiver of a 7-year-old female described, "So on the extreme... she was hitting my husband. But like I said it was just really just once. Then on the other end, it's just her face gets red and... she'll get right close to you, it's almost like her voice gets really deep and she'll say, 'I don't want to do that."'

Anxiety was reported for all twelve participants but varied in frequency from every day to a few times a week. Anxiety behaviors included skin picking, repetitive questions and fidgeting, and causes of anxiety were most often reported as food- or routine-related (food or nonfood). As described by the caregiver of a 14-yearold male: "He starts darting around a lot, he's sort of looking around anxiously and everything. He starts to tap his, shake his leg, so, like, not necessarily tap his foot but sort of tapping his whole leg and... the number of questions goes up and his volume and his voice just goes up."

All 12 caregivers noted hyper-focusing, but the targets varied. Two caregivers said their child hyper-focused on activities, three said their child hyper-focused on topics, and seven reported their child's hyper-focusing targeted multiple aspects of life (activities, people, and topics).

The ability to cope with non-food-related change was reportedly good for three trial participants. Nine caregivers reported issues with their child's ability to cope with change, as described by the caregiver of a 7-year-old female: "She doesn't usually do well with change at all. Like sometimes if I know something is going to change we usually try and tell her ahead of time.
Table 3: Summary of baseline aspects of daily life.

\begin{tabular}{|l|l|}
\hline Aspects of Daily Life & Subjects n/N (\%) \\
\hline Lacking age-appropriate independence & $7 / 12(58)$ \\
\hline Exercise intolerance & $10 / 12(83)$ \\
\hline Extensive planning required for daily life & $10 / 12(83)$ \\
\hline $\begin{array}{l}\text { Caregiver avoidance of certain events, } \\
\text { activities, or situations }\end{array}$ & $12 / 12(100)$ \\
\hline Excessive daytime sleepiness & $7 / 12(58)$ \\
\hline Wakes up during the night & $5 / 12(42)$ \\
\hline Wakes up earlier than expected & $3 / 10(30)$ \\
\hline
\end{tabular}

That's something is going to change...sometimes that works for her and sometimes it doesn't."

When caregivers were asked to describe the most-problematic non-food-related behavior, three cited dealing with changes in routine. For the remaining nine, there were a variety of responses, including tantrums, following directions, stealing, anxiety, hyper-focus, rigid and argumentative social interactions, and aggression.

\section{Aspects of Daily Life}

A summary of selected aspects of daily life can be found in Table 3 and in Figure 4. In Table 3, N was 10 for 'wakes up earlier than expected' because two caregivers did not address this topic.

Some aspects of daily life were present for most or all of the participants, while others were less prevalent. However, even in the more prevalent aspects of daily life, their nature varied. More than half of the caregivers (7 of 12) reported their children exhibited less independence than expected for their age. Those exhibiting no independence ( 3 of 12) were young (ages 7, 8 and 9), while older trial participants (ages 14-25) were more likely to be described as having some measure of independent functioning. Notably, seven of the caregivers noted their child's limited independence was by design - families ensured constant supervision. Also, three trial participants lacking age-appropriate independence were described as having developmental disabilities that limited independence. Only one caregiver reported a high-level of functional independence; in that case, the 11-year-old girl was able to autonomously manage all her self-care in an age-appropriate way. One caregiver reported her 25 -year-old son was affected by his perceived difference in independence relative to his non-PWS peers, describing, "He sees them becoming independent, living alone, traveling, doing all these things that he is not able to do. And so that's very frustrating to him."

Sleep issues were described by 10 caregivers. Of these 10 participants, all ages and genders were represented, and all experienced at least some daytime sleepiness. Additionally, five caregivers reported problematic nighttime waking by the participant, and three 


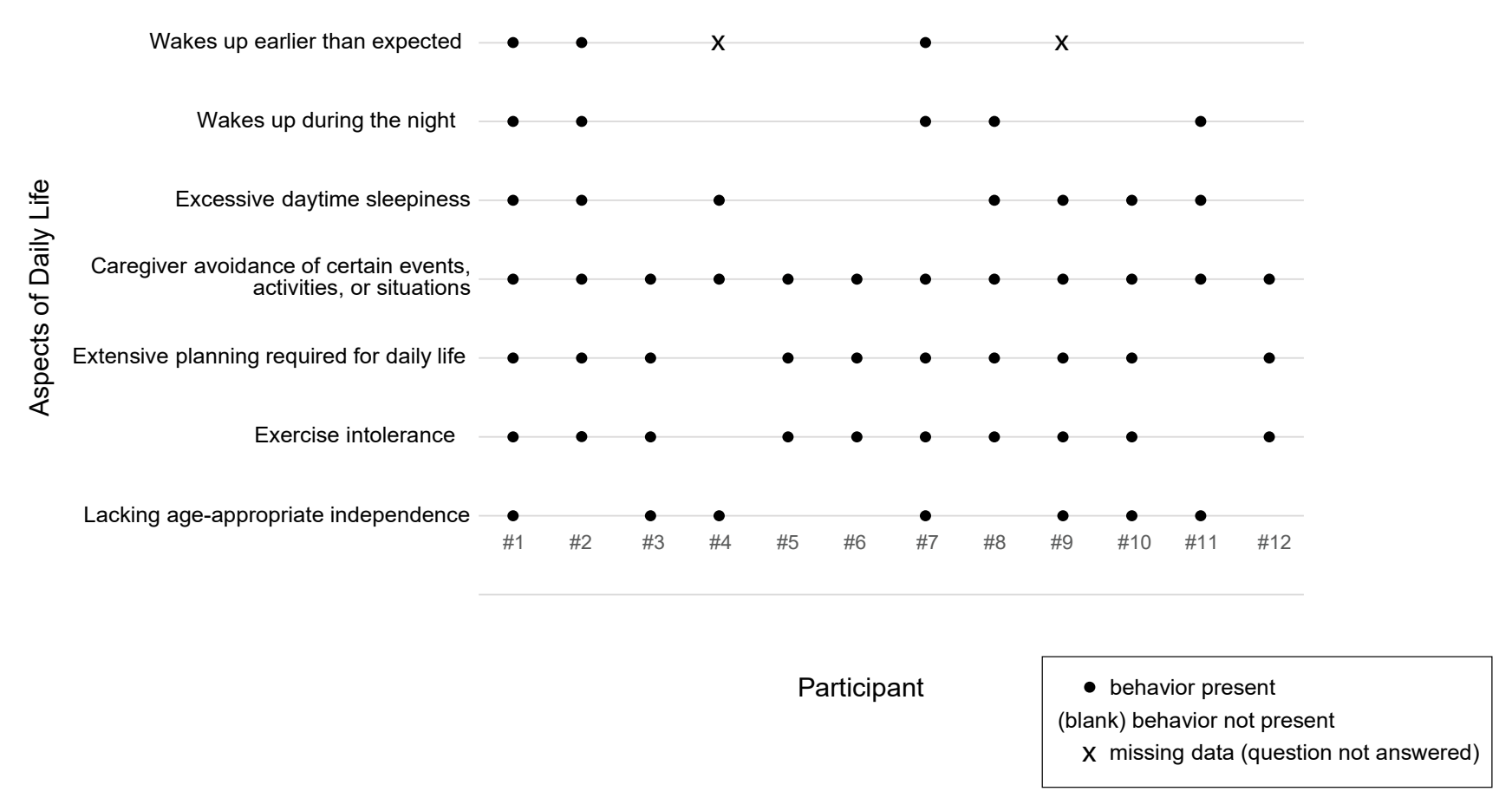

Figure 4: Daily Life by Participant at Baseline.

caregivers reported the participant rising earlier than expected. Sleep issues were also mentioned in the context of overall behavior, with many caregivers citing the participant's tiredness as a direct contributor to other problematic behaviors, as described by the caregiver of a 6-year-old female: "Because when she gets good sleep, I don't see the other behaviors. So it's almost like the fatigue is a symptom of not getting enough sleep. Her tantrums are a symptom of not getting good sleep."

The most problematic aspects of daily life varied and included food-related behavior, sleep issues, social functioning, cognitive rigidity/argumentativeness, and unwillingness to exercise.

\section{Discussion}

We report the methodology for developing an interview guide, the conduct of baseline qualitative interviews with caregivers of PWS patients, and the analysis from the baseline qualitative interview data. The findings indicated some behaviors and symptoms are present in a subset of participants with PWS while other common PWS behavioral characteristics can present with different manifestations or intensities. Behavior during meals varied widely among trial participants, as did the degree of their independence. Discussions about food occurred in all families but ranged from productive interactions to aggressive confrontations. Additionally, the baseline data revealed the importance of context in interpreting behavioral data. For example, all 12 caregivers indicated PWS individuals would steal or sneak food, but the frequency of this behavior varied and was highly affected by degree of oversight and availability of food in the immediate environment. Lastly, when asked about the most problematic behavior within each category (food-related behavior, general behavior, and daily life), answers varied widely. Findings from the baseline data confirmed the conclusions drawn from the literature, most notably the degree of heterogeneity in the population and the impact of the historical and immediate context on the behavior and symptoms of an individual with PWS.

Capturing information about the heterogeneity of the study population qualitatively allowed caregivers to use their own vocabulary and framework to describe their child's behavior and function. The interviews enabled the capture of a greater degree of nuance in the description of trial participants, their home environment, and factors that affected their behavior.

In clinical trials studying rare diseases, a tension exists between adequately powering studies and limiting the heterogeneity of the study population. In PWS, heterogeneity is multi-faceted, affected by genetics, patient age, the environment the person with PWS was raised in, and the immediate circumstances around the time of data capture; additional factors may also contribute. This heterogeneity may affect the sensitivity of the population to specific measures. For example, a person with PWS raised with locked garbage since infancy may never try to break into the trash, and a child whose parents never negotiate about food may eventually stop trying, both topics of questions on the HQ-CT. In addition, concepts that might be meaningful to families may not be reflected in the chosen outcome measure. For example, caregivers in the baseline interviews mentioned their children's ability to feel satiety, their families' ability to eat out, and their children's ability to make good food choices were meaningful behaviors that are excluded from the HQ-CT. 
The purpose of this sub-study was to obtain individual experience data useful in providing context to the more quantitative approaches used to determine efficacy in the DCCR Phase III program. Future analysis of longitudinal data will determine whether families report specific changes in trial participants with PWS at a higher rate following a change in treatment status in both the randomized portion of the study and the OLE.

\section{Limitations}

The limitations of the data include the small number of caregivers enrolled in this sub-study. The sub-study was started 15 months after the initiation of the trial so the recruitment time for the sub-study was limited. In addition, the sample may have been biased by the optin structure of the sub-study, with caregivers choosing to participate in the sub-study representing certain family or participant behaviors or characteristics.

\section{Conclusion}

To fully understand a potential treatment effect in PWS, it is crucial to understand the specifics of each participant's behavior, environment, and circumstance, and examine any changes through the specific lens of that person. Using a mixed methods approach, qualitative interviews complement standard validated outcome measures as a strategy for fully capturing, evaluating, and understanding complicated behaviors and changes to behaviors that may result from a therapeutic intervention, especially for a rare syndrome with a limited and heterogenous population.

\section{Acknowledgements}

The authors would like to thank the families that participated in this study. This study was conducted with funding from Soleno Therapeutics, Inc. A Bhatnagar, K Yen, and $P$ Hirano are employees of Soleno Therapeutics. PS. is involved in clinical research funded by Soleno Therapeutics, Inc. and Millendo Therapeutics, Inc.

JM has received research funding from Soleno Therapeutics, Levo Therapeutics, Harmony, Rhythm Pharmaceuticals, and is a consultant for Consyance. $\mathrm{AH}$. is a local PI in the UK arm of the Destiny clinical trial funded by Soleno, an adviser to Novo Nordisk, Radius Health and Saniona on their trials, and is President of the International PWS Organisation.

\section{Statement of Author's Contribution}

M Leffler, A Bhatnagar, $K$ Yen, and $P$ Hirano conceived and planned the study. $M$ Leffler, and $M$ Hefner performed the data collection. M Leffler, $\mathrm{S}$ Elder, S Bolding and $\mathrm{K}$ Davis performed the data analysis and interpretation. M Leffler, S Elder, S Bolding and K Davis drafted the article. All authors helped shape the research, provided critical feedback, and contributed to the final manuscript. All authors approved the final version of the manuscript for publication.

\section{References}

1. Bittel DC, Butler MG (2005) Prader-Willi syndrome: Clinical genetics, cytogenetics and molecular biology. Expert Rev Mol Med 7: 1-20.

2. Miller JL (2012) Approach to the child with Prader-Willi Syndrome. J Clin Endocrinol Metab 97: 3837-3844.

3. Goldstone AP, Holland AJ, Hauffa BP, Hokken-Koelega AC, Tauber M (2008) Recommendations for the diagnosis and management of Prader-Willi Syndrome. J Clin Endocrinol Metab 93: 4182-4197.

4. Crawford SR, Brown TM, Fehnel SE, Doward LC, Nelson L, et al. (2015) The International development of the modified hyperphagia questionnaire. Poster presented at ISPOR 18th Annual European Congress, Milan Italy.

5. National Institute of Health. Prader-Willi Syndrome.

6. Cassidy SB, McCandless SE (2021) Prader-Willi syndrome. In: Cassidy SB, Allanson JE, Management of Genetic Syndromes Hoboken. NJ: Wiley-Liss.

7. Duis J, Wattum PJ van, Scheimann A, Parisa Salehi, Elly Brokamp, et al. (2019) A multidisciplinary approach to the clinical management of Prader-Willi syndrome. Mol Genet Genomic Med 7: e514.

8. National Organization for Rare Diseases. Rare Disease Directory: Prader-Willi Syndrome.

9. Forster JL, Gourash LM (2005) Prader-Willi Syndrome: A primer for psychiatrists. Pittsburgh Partnership.

10. Ho AY, Dimitropoulos A (2010) Clinical management of behavioral characteristics of Prader-Willi syndrome. Neuropsychiatr Dis Treat 6: 107-118.

11. Gantz MG, Andrews SM, Wheeler AC (2020) Food and non-food-related behavior across settings in children with Prader-Willi Syndrome. Genes 11: 204-217.

12. (2019) Food and Drug Administration. Patient-focused drug development: Methods to identify what is important to participants guidance for industry, Food and Drug Administration staff, and other stakeholders. 